\title{
Full-intensity waveform inversion
}

\author{
Yike Liu', Bin $\mathrm{He}^{1}$, Huiyi Lư ${ }^{1}$, Zhendong Zhang², Xiao-Bi Xie ${ }^{3}$, and Yingcai Zheng ${ }^{4}$
}

\begin{abstract}
Many full-waveform inversion schemes are based on the iterative perturbation theory to fit the observed waveforms. When the observed waveforms lack low frequencies, those schemes may encounter convergence problems due to cycle skipping when the initial velocity model is far from the true model. To mitigate this difficulty, we have developed a new objective function that fits the seismic-waveform intensity, so the dependence of the starting model can be reduced. The waveform intensity is proportional to the square of its amplitude. Forming the intensity using the waveform is a nonlinear operation, which separates the original waveform spectrum into an ultra-low-frequency part and a higher frequency part, even for data that originally do not have low-frequency contents. Therefore, conducting multiscale inversions starting from ultra-low-frequency intensity data can largely avoid the cycle-skipping problem. We formulate the intensity objective function, the minimization process, and the gradient. Using numerical examples, we determine that the proposed method was very promising and could invert for the model using data lacking low-frequency information.
\end{abstract}

\section{INTRODUCTION}

Full-waveform inversion (FWI) can be used to reconstruct subsurface model parameters such as the velocity, density, and other medium properties. The FWI is usually cast as a global nonlinear optimization problem to minimize an objective function, which is nonlinear with respect to medium parameters. To solve the FWI problem, one usually starts from an initial model and solve it in an iterative fashion. In each step, the process linearizes the nonlinear problem and tries to find the model perturbation such that it minimizes the misfit between observed waveform data and synthetic records calculated using the updated model by adding the model perturbation to the starting model (Lailly, 1983; Tarantola, 1984). The linearization is equivalent to the Born single scattering (Tarantola, 2005; Wu and Zheng, 2014), which provides accurate approximation in the wavefield calculation when the heterogeneities are small-scale, weak in perturbation, or the incident-wave frequency is low. When the initial velocity model is close to the true velocity model, the iteration process can readily converge to a global optimal solution, usually yielding a model with higher spatial resolution compared with conventional traveltime tomography or migration velocity analysis (Virieux and Operto, 2009). On the other hand, an inaccurate initial velocity model and lack of low-frequency contents in the data can often cause the objective function to converge to local minima due to cycle skipping.

To alleviate this cycle-skipping difficulty, many multiscale FWI strategies and crosscorrelation type objective functions were proposed (e.g., Bunks et al., 1995; Pratt, 1999; Ravaut et al., 2004; Sirgue and Pratt, 2004; Shen and Symes, 2008; Leeuwen and Mulder, 2010; Plessix et al., 2010; Xu et al., 2012; Zhou et al., 2012; Brittan et al., 2013). FWI based on such techniques implement a sequential inversion scheme by first using the lowest available frequency to recover low-wavenumber (i.e., large-scale) model structures, followed by using the high-frequency data to invert for high-wavenumber (i.e., fine-scale) structures. Baeten et al. (2013) demonstrate that the frequency content less than $2 \mathrm{~Hz}$ is needed to properly recover the low-wavenumber structures. However, most of the current dataacquisition systems do not provide such low-frequency information.

An accurate initial model building is a key component in FWI. Alkhalifah and Choi (2014) and Zhang et al. (2015) propose a workflow to use traveltime tomography and multiple objective functions to obtain a low-wavenumber initial model for the subsequent FWI. However, limited by the high-frequency asymptotic approximation,

\footnotetext{
Manuscript received by the Editor 16 October 2017; revised manuscript received 11 June 2018; published ahead of production 30 August 2018; published online 23 October 2018 .

${ }^{1}$ Institute of Geology and Geophysics, Key Laboratory of Petroleum Resource Research, Chinese Academy of Sciences, Beijing, China. E-mail: ykliu@ mail .iggcas.ac.cn (corresponding author); hebin@mail.iggcas.ac.cn; lhy061981@163.com.

${ }^{2}$ King Abdullah University of Science and Technology, Thuwal, Saudi Arabia. E-mail: zhendong.zhang@kaust.edu.sa.

${ }^{3}$ University of California at Santa Cruz, Santa Cruz, California, USA. E-mail: xxie@ucsc.edu.

${ }^{4}$ University of Houston, Houston, Texas, USA. E-mail: yzheng12@uh.edu.

(C) 2018 Society of Exploration Geophysicists. All rights reserved.
} 
this ray-based tomography encounters difficulties in a complex velocity model. The wave-equation-based traveltime tomography can accommodate the multipathing problem by incorporating finite-frequency wave propagation (Luo and Schuster, 1991; Clément et al., 2001; Almomin and Biondi, 2012; Xu et al., 2012; Ma and Hale, 2013). Other ray-based or wave-equation-based methods (Zhang and Wang, 2009; Biondi and Almomin, 2013; Zhang et al., 2015; Luo, et al., 2016) were proposed to estimate a smooth large-scale initial velocity model. These methods emphasize the usefulness of the traveltime information more than the amplitude information of the waves. Instead of having separate methodologies for the initial model building and the following FWI, much effort has been invested on integrating them into one coherent process. Shin and Cha (2009) modulate seismic signals to much lower frequencies in the LaplaceFourier domain to reconstruct large-scale velocity anomalies. The envelope-inversion method extracts very low-frequency information from the data and uses a demodulation operator to recover smooth background velocity (Bozdag et al., 2011; Wu et al., 2014; Luo and Wu, 2015). Alkhalifah and Wu (2015), Xie (2015), and Bharadwaj et al. (2016) propose a multiscale inversion strategy that uses the scattering angle information to control the wavenumber contents in the velocity-model updating.

To avoid cycle skipping, the direct waveform inversion (DWI) method (Liu and Zheng [2015], for 2D development) can be used to invert the waveform data without using a global initial model; furthermore, with this method, no iteration is required for model updating. Starting from the source-receiver region and using time-space causality of the wavefield, the DWI builds the model outward recursively by fitting the earliest part of the waveforms. Choi and Alkhalifah (2017) develop a method using an energybased objective function to improve the efficient calculation of the gradient and cycle skipping.

In this paper, we propose a new waveform-inversion method, called full-intensity waveform inversion (FIWI), which is based on the waveform intensity. We can show that, by taking the intensity of the waveform, the frequency content of the original waveform is decomposed into a low- and a high-frequency part. The former contains the frequency content, which is lower than that in the original waveform. Using this information to estimate the long-wavelength velocity model can be less affected by cycle skipping. For this purpose, we developed an objective function based on the difference between the intensities of modeled and observed data. We then derive its gradient operator with respect to the velocity-model perturbations and propose a new type least-squares minimization procedure toward velocity model updating. The intensity-based FWI approach can be divided into two steps: first, using the low-frequency intensity data to obtain a large-scale background velocity model and, second, taking this model as the starting model and using the conventional waveform FWI to obtain a high-resolution final model. We present numerical examples to validate our proposed

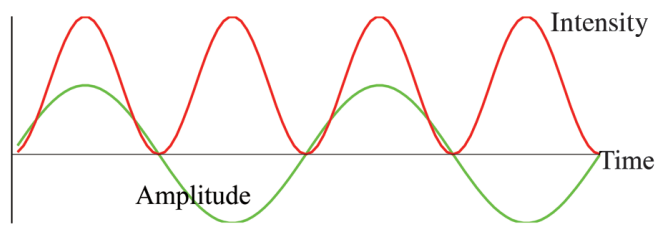

Figure 1. The blue line is the sine function of a wave time series, and the red line is the square of its amplitude denoted as intensity. method, and we demonstrate that FIWI can successfully recover long-wavelength, strong velocity perturbations using waveforms with no low-frequency content.

\section{METHODOLOGY}

\section{Review of conventional full-waveform inversion}

The conventional FWI seeks to minimize the misfit between observed and modeled data by finding a subsurface model. It can be formulated as an optimization process:

$$
\min E(\mathbf{m})=\frac{1}{2} \sum_{s} \sum_{r} \int_{t}\left[d_{c}\left(\mathbf{x}_{r}, t ; \mathbf{x}_{s} \mid \mathbf{m}\right)-d_{o}\left(\mathbf{x}_{r}, t ; \mathbf{x}_{s}\right)\right]^{2} d t,
$$

where $E$ is the misfit function, $\mathbf{m}$ represents the subsurface velocity model to be inverted for, $d_{o}\left(\mathbf{x}_{r}, t ; \mathbf{x}_{s}\right)$ is the observed time $(t)$ domain waveform data at receiver $\mathbf{x}_{r}$ due to a source at $\mathbf{x}_{s}$, and $d_{c}\left(\mathbf{x}_{r}, t ; \mathbf{x}_{s} \mid \mathbf{m}\right)$ is the modeled waveform data using model $\mathbf{m}$. The goal is finding an $\mathbf{m}$ that makes $d_{c}$ best fit the observed data $d_{o}$. Due to its nonlinear feature, equation 1 is usually linearized based on the perturbation theory and solved iteratively. However, if the initial model deviated severely from the true velocity and the data lacks of low-frequency contents, the above-mentioned linearized inversion process may encounter convergence problems. For a multiscale implementation, the iteration starts from the lowest-frequency because low-frequency data can tolerant larger errors in the initial model. However, the low-frequency components are not always available. For example, a conventional acquisition system rarely provides information at less than $2 \mathrm{~Hz}$. To mitigate this difficulty, we introduce FIWI.

\section{The frequency-domain feature of the wave intensity}

The amount of energy passing through a unit area per unit of time in a specified direction is called the intensity of the wave. Unlike pressure or the displacement wavefield, the wave intensity has quite different frequency characteristics. The wave intensity is proportional to the square of its amplitude (Born and Wolf, 1999), as shown in Figure 1.

Consider two time series $u(t)$ and $v(t)$, their product $w(t)=u(t)$ - $v(t)$ has the Fourier spectrum expressed in the convolution form

$$
W(\omega)=\int_{-\infty}^{\infty} U(\xi) V(\omega-\xi) d \xi
$$

where $U$ and $V$ are the Fourier transforms of $u(t)$ and $v(t)$, respectively, $t$ is the time, $\omega=2 \pi f$ is the angular frequency, and $f$ is the frequency in hertz. If $u(t)=v(t)$, then $w(t)$ denotes the intensity. The convolution of spectra causes the frequency content split into a low- and a high-frequency band. Consider a simple example in which $u(t)$ is composed of two harmonic signals $u(t)=$ $\cos \left(2 \pi f_{1} t\right)+\cos \left(2 \pi f_{2} t\right)$ where $f_{1}=20 \mathrm{~Hz}$ and $f_{2}=25 \mathrm{~Hz}$. The Fourier transforms of $u(t)$ and its intensity $w(t)$ are illustrated in Figure $2 \mathrm{a}$ and $2 \mathrm{~b}$, respectively. Although the original signal only has frequency components at 20 and $25 \mathrm{~Hz}$, the nonlinear square operation separates frequency components into a low-frequency group ( 0 and $5 \mathrm{~Hz})$ and a high-frequency group (40, 45, and $50 \mathrm{~Hz}$ ). If $f_{1}$ and $f_{2}$ are very close, strong low-frequency components can be 
obtained at near-zero frequency. We note that this low-frequency content is for the intensity and not for the waveform. In a slightly more complicated example, consider a band-limited signal

$$
u(t)=\sum_{i=0}^{N} e^{-\frac{1}{2 c^{2}}\left(\frac{i}{2}-f_{p}\right)^{2}} \cos \left[2 \pi\left(f_{\min }+\frac{i}{2}\right) t\right]
$$

which is composed of 41 harmonic components starting from $f_{\min }=$ $10 \mathrm{~Hz}$ and has a Gaussian-shaped spectrum with a dominant frequency at $f_{p}=20 \mathrm{~Hz}$, and a characteristic bandwidth $\sqrt{2} c=10$. The Fourier spectra of $u(t)$ and its intensity are illustrated in Figure 3a and $3 \mathrm{~b}$, respectively. The intensity spectrum is again split into two frequency bands, with the one located at ultra-low frequencies near zero, and the other located between 30 and $50 \mathrm{~Hz}$ with a dominant frequency at $2 f_{p}$. The largest amplitude in the spectrum is located at the low frequencies. Between the low- and high-frequency bands, there is a gap, which is approximately the frequency band of the original waveform signal, which is shifted to the low and high frequencies during the intensity calculation. Now, the $u(t)$ and its intensity are composed of three frequency bands isolated from each other, e.g., the ultra-low-frequency band with $0-10 \mathrm{~Hz}$ associated with intensity, the original data with $10-30 \mathrm{~Hz}$ band, and the higher-frequency band related to the intensity at $30-50 \mathrm{~Hz}$. Using the intensity characteristics for the ultra-low frequencies, we can update a velocity from 0 up to $10 \mathrm{~Hz}$ by multiscale FWI using intensity and then we can implement subsequent steps by multiscale FWI or FWI only using the original waveform data expressed in equation 3 .

\section{FIWI}

We propose to minimize the misfit between intensities of the observed and synthetic data. An intensity object function can be defined as

$$
\begin{aligned}
\min E(\mathbf{m})= & \frac{1}{4} \sum_{s} \sum_{r} \int\left(d_{c}^{2}\left(\mathbf{x}_{r}, t ; \mathbf{x}_{s}\right)\right. \\
& \left.-d_{o}^{2}\left(\mathbf{x}_{r}, t ; \mathbf{x}_{s}\right)\right)^{2} d t
\end{aligned}
$$

where $d_{c}^{2}\left(\mathbf{x}_{r}, t ; \mathbf{x}_{s}\right)$ and $d_{o}^{2}\left(\mathbf{x}_{r}, t ; \mathbf{x}_{s}\right)$ are the intensities of the synthetic and observed data, respectively, and the factor $1 / 4$ is introduced for subsequent simplifications. A model perturbation of equation 4 is given using Taylor expansion by minimizing the objective function:

$$
\begin{aligned}
& \delta \mathbf{m}=\frac{\partial E\left(\mathbf{m}_{\mathbf{0}}\right)}{\partial \mathbf{m}}, \\
& =\sum_{s} \sum_{r} \frac{\partial d_{c}}{\partial \mathbf{m}} \int d_{c}\left[d_{c}^{2}\left(\mathbf{x}_{r}, t ; \mathbf{x}_{s}\right)\right. \\
& \left.\quad-d_{o}^{2}\left(\mathbf{x}_{r}, t ; \mathbf{x}_{s}\right)\right] d t .
\end{aligned}
$$

Setting $I_{c}=d_{c}^{2}\left(\mathbf{x}_{r}, t ; \mathbf{x}_{s}\right)$ and $I_{o}=d_{o}^{2}\left(\mathbf{x}_{r}, t ; \mathbf{x}_{s}\right)$, and

$$
d I\left(\mathbf{x}_{r}, \mathbf{x}_{s}, t\right)=I_{c}-I_{o}
$$

is called the intensity residual. We then have

$$
\delta \mathbf{m}=\frac{\partial E\left(\mathbf{m}_{\mathbf{0}}\right)}{\partial \mathbf{m}}=\sum_{s} \sum_{r} \int_{t} S\left(\mathbf{x}_{r}, t ; \mathbf{x}_{s}\right) \frac{\partial d_{c}\left(\mathbf{x}_{r}, t ; \mathbf{x}_{s}\right)}{\partial \mathbf{m}} d t,
$$

where the first-order derivative of the wavefield with respect to the model parameters $\partial d_{c}\left(\mathbf{x}_{r}, t ; \mathbf{x}_{s}\right) / \partial \mathbf{m}$ is referred to as the Frechét derivative and

$$
S\left(\mathbf{x}_{r}, t ; \mathbf{x}_{s}\right)=d I\left(\mathbf{x}_{r}, t ; \mathbf{x}_{s}\right) d_{c}\left(\mathbf{x}_{r}, t ; \mathbf{x}_{s}\right)
$$

is the adjoint source that can be back propagated into the subsurface. The model perturbation is searched in the opposite direction of the steepest ascent, i.e., the gradient of the misfit function at point $\mathbf{m}_{\mathbf{0}}$. FIWI extrapolates the product of the intensity residual and the synthetic waveform $d_{c}\left(\mathbf{x}_{r}, t ; \mathbf{x}_{s}\right)$, rather than the wavefield residuals, to the subsurface. If the traveltime difference between the synthetic and observed data is larger than the half period of the lowest-frequency signal in the data, conventional FWI may encounter convergence difficulty due to cycle skipping. The proposed intensity method can mitigate the cycle skipping because it contains ultra-low-frequency contents.

Near-offset traces usually have much larger amplitudes compared with those at far offsets, causing the updating mostly limited at the shallower part of the model. To balance their contributions, we normalize the intensities by modifying equation 4 into

$$
\min E(\mathbf{m})=\frac{1}{4} \sum_{s} \sum_{r} \int_{t} d I_{\mathrm{norm}}\left(\mathbf{x}_{r}, t ; \mathbf{x}_{s}\right)^{2},
$$

where
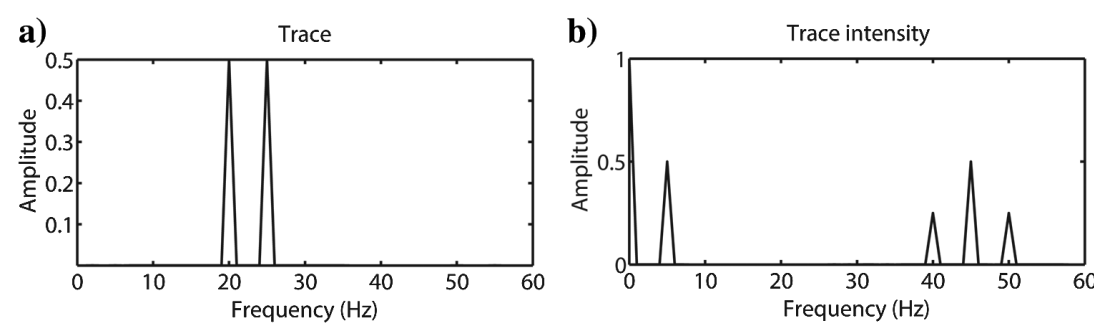

Figure 2. (a) Spectrum of a sine-based function composed of two frequencies with $f_{1}=20$ and $f_{2}=25 \mathrm{~Hz}$ and (b) the spectrum of the product of the sine function consists of two group ranges, e.g., low- and high-frequency areas.
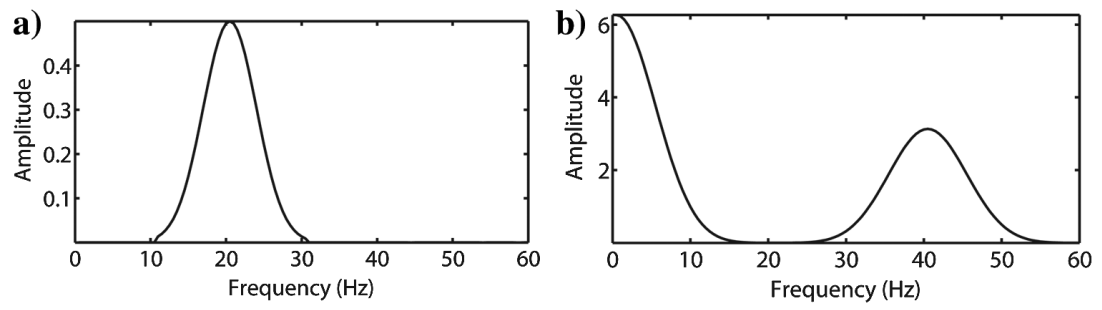

Figure 3. (a) The spectrum of signal $u(t)$, in which the frequencies range from 10 to $30 \mathrm{~Hz}$ with dominant frequency at $20 \mathrm{~Hz}$. (b) The spectrum of the intensity of $u(t)$, which has a low-frequency part corresponding to the frequencies from 0 to $20 \mathrm{~Hz}$ and a high-frequency part with frequencies from 20 to $60 \mathrm{~Hz}$. There exists a gap at the spectrum of the intensity between 10 and $30 \mathrm{~Hz}$ - this region corresponds to the frequencies of the original data. 
a)

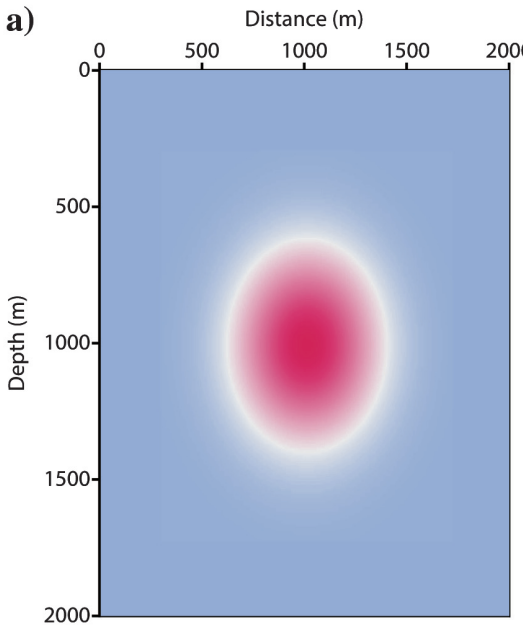

c)
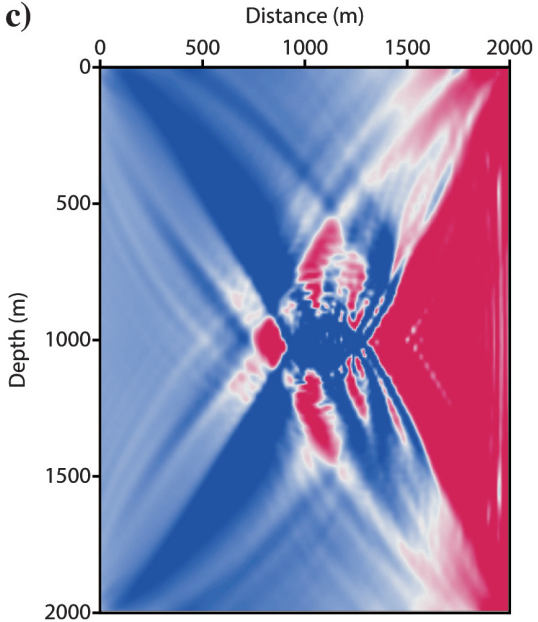

b)

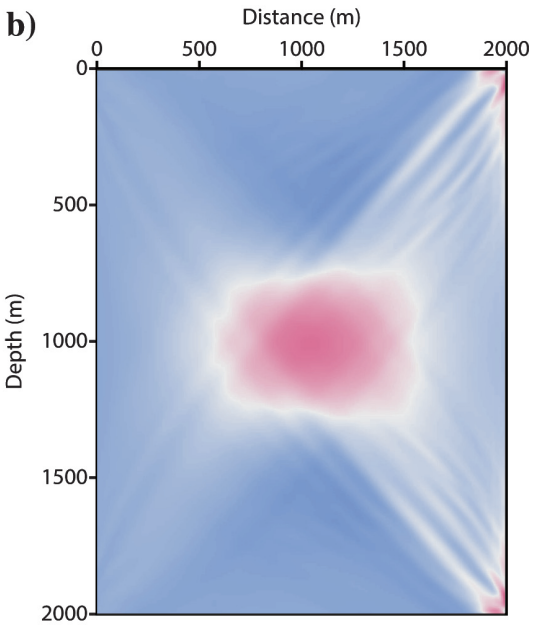

d)

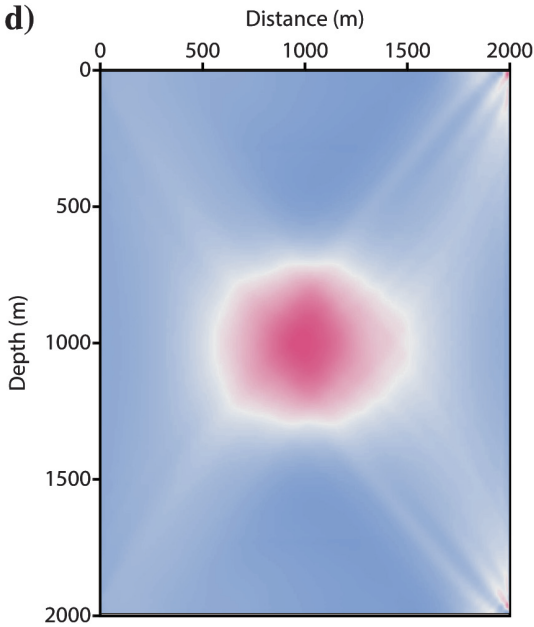

Figure 4. (a) True model with a Gaussian-ball velocity anomaly, (b) Gaussian-ball perturbation inverted by FIWI with intensity data using multiscale from $0-0-3-6 \mathrm{~Hz}$ after three iterations, $0-0-6-9 \mathrm{~Hz}$ after two iterations, $0-0-9-12 \mathrm{~Hz}$ after 74 iterations, $0-0-12-$ $15 \mathrm{~Hz}$ after one iteration. (c) Results from conventional FWI using the data from 15-20$30-40 \mathrm{~Hz}$, which fails to recover the Gaussian ball. (d) The Gaussian-ball velocity model recovered using the bandwidth $10-15-30-40 \mathrm{~Hz}$ with conventional FWI after 100 iterations.

a)
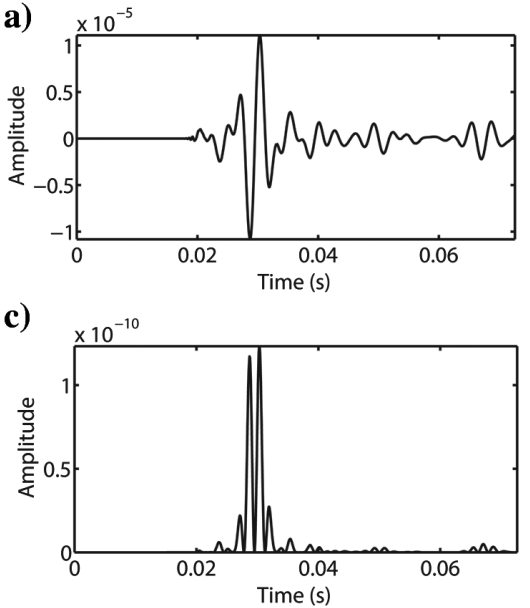

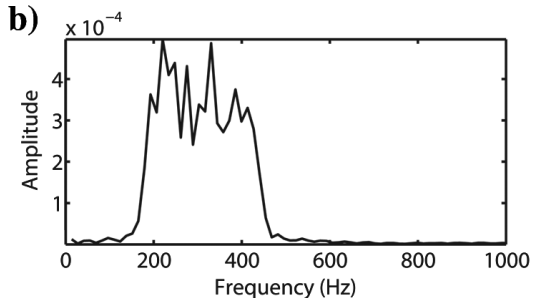

d)

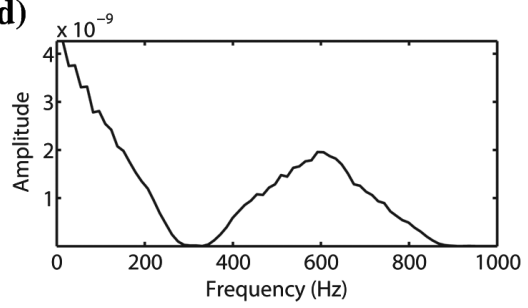

Figure 5. A trace, intensity, and their frequencies of crosswell data. (a) A crosswell trace, (b) the frequency spectrum of the trace, (c) intensity of the trace, and (d) the frequency spectrum of the trace intensity.

$$
d I_{\mathrm{norm}}\left(\mathbf{x}_{r}, \mathbf{x}_{s}, t\right)=\frac{d_{c}^{2}\left(\mathbf{x}_{r}, t ; \mathbf{x}_{s}\right)}{\bar{P}_{c}\left(\mathbf{x}_{r} ; \mathbf{x}_{s}\right)}-\frac{d_{o}^{2}\left(\mathbf{x}_{r}, t ; \mathbf{x}_{s}\right)}{\bar{P}_{o}\left(\mathbf{x}_{r} ; \mathbf{x}_{s}\right)}
$$

is the normalized intensity residual and the denominators

$$
\begin{aligned}
& \bar{P}_{c}\left(\mathbf{x}_{r} ; \mathbf{x}_{s}\right)=\frac{1}{T} \int_{\tau=0}^{T} d_{c}^{2}\left(\mathbf{x}_{r}, \tau ; \mathbf{x}_{s}\right) d \tau, \\
& \bar{P}_{o}\left(\mathbf{x}_{r} ; \mathbf{x}_{s}\right)=\frac{1}{T} \int_{\tau=0}^{T} d_{o}^{2}\left(\mathbf{x}_{r}, \tau ; \mathbf{x}_{s}\right) d \tau
\end{aligned}
$$

are the average power of the individual traces, which normalize the observed and synthesized intensities, eliminating the amplitude problem. The gradient of the objective function using the normalized intensity can be derived as (see Appendix A for details)

$$
\begin{aligned}
\delta \mathbf{m} & =\frac{\partial E(\mathbf{m})}{\partial \mathbf{m}} \\
& =\sum_{s} \sum_{r}\left[\int_{0}^{T} S_{\mathrm{vrc}}\left(\mathbf{x}_{r}, t ; \mathbf{x}_{s}\right) \frac{\partial d_{c}\left(\mathbf{x}_{r}, t ; \mathbf{x}_{s}\right)}{\partial \mathbf{m}} d t\right],
\end{aligned}
$$

where

$$
\begin{aligned}
& S_{\mathrm{vrc}}\left(\mathbf{x}_{r}, t ; \mathbf{x}_{s}\right)=\left(d I_{\mathrm{norm}}\left(\mathbf{x}_{r}, t ; \mathbf{x}_{s}\right)\right. \\
& \left.-\frac{\frac{1}{T} \int_{\tau=0}^{T} d I_{\mathrm{norm}}\left(\mathbf{x}_{r}, \tau ; \mathbf{x}_{s}\right) d_{c}^{2}\left(\mathbf{x}_{r}, \tau ; \mathbf{x}_{s}\right) d \tau}{\bar{P}_{c}\left(\mathbf{x}_{r} ; \mathbf{x}_{s}\right)}\right) \\
& \quad \times \frac{d_{c}\left(\mathbf{x}_{r}, t ; \mathbf{x}_{s}\right)}{\bar{P}_{c}\left(\mathbf{x}_{r} ; \mathbf{x}_{s}\right)}
\end{aligned}
$$

is the adjoint source. Note that equations 12 and 7 are very similar. The difference lies in the adjoint sources $S\left(\mathbf{x}_{r}, t ; \mathbf{x}_{s}\right)$ and $S_{\mathrm{vrc}}\left(\mathbf{x}_{r}, t ; \mathbf{x}_{s}\right)$ (i.e., equations 8 and 13). After we introduce the normalized intensity difference $d I_{\text {norm }}$ to replace the intensity difference $d I$, an additional term, i.e., the second term in the bracket of equation 13, needs to be added.

For addressing and referring to the definition of the $I_{c}$ and $I_{o}$ in equation 6 , the second term in the bracket in equation 13 can be expressed as

$$
\begin{gathered}
\operatorname{Cross} I_{o} I_{c}=\frac{1}{\bar{P}_{c}\left(\mathbf{x}_{r} ; \mathbf{x}_{s}\right)}\left(\left(\frac{1}{T} \int_{0}^{T} I_{c}^{2}\left(\mathbf{x}_{r}, \tau ; \mathbf{x}_{s}\right) d \tau\right.\right. \\
\left.-\frac{1}{T} \int_{0}^{T} I_{o}\left(\mathbf{x}_{r}, \tau ; \mathbf{x}_{s}\right) I_{c}\left(\mathbf{x}_{r}, \tau ; \mathbf{x}_{s}\right) d \tau\right) .
\end{gathered}
$$

The first term in equation 14 is the zero-lag autocorrelation of the modeled intensity, and the second term is the zero-lag crosscorrelation between the modeled and the observed intensity. The gradient in equation 13 attempts to update 
the model by combining the intensity difference and the correlation between the observed data and the modeled intensities. This facilitates the objective function to converge to the global minimum. Our adjoint source $S_{\mathrm{vrc}}\left(\mathbf{x}_{r}, t ; \mathbf{x}_{s}\right)$, which estimates the intensity residual between the observed data and the synthetic data, significantly mitigate cycle skipping and avoid the local minima problem.

\section{NUMERICAL EXAMPLES}

\section{Gaussian-ball model}

We demonstrate a crosswell experiment with a Gaussian anomaly to test the ability using our FIWI as opposed to the conventional FWI (Tarnatola, 1984; Bunks et al., 1995). A Gaussian-ball velocity anomaly with velocity of $2200 \mathrm{~m} / \mathrm{s}$ is located at the center of a homogeneous medium. The homogeneous medium is characterized by a constant velocity of $2000 \mathrm{~m} / \mathrm{s}$ (Figure 4a). In the crosswell geometry, 200 seismic sources are distributed along the left well spaced at a $10 \mathrm{~m}$ interval and 200 geophones are inside the right well spaced at the same interval. We use two Butterworth wavelets (Zhou and McMechan, 1999) to generate two sets of data for different frequency intervals $10-15-30-40$ and $15-20-30-40 \mathrm{~Hz}$, respectively. We first test FIWI and the conventional FWI to the waveform data set without information of less than $15 \mathrm{~Hz}$. Although the data with frequencies 15-20-30-40 Hz do not have contents less than $15 \mathrm{~Hz}$, the intensity has very-lowfrequency components. A band-pass filter is applied to obtain intensity data that contain $0-0-3-6,0-0-6-9,0-0-9-12$, and $0-0-12-$ $15 \mathrm{~Hz}$ data associated with the original data 15-20-30-40 Hz. FIWI implementation starts from an initial homogeneous velocity with multiscale procedure from low to high frequencies. The Gaussian-ball perturbation with intensity data using the multiscale procedure from $0-0-3-6 \mathrm{~Hz}$ after three iterations, $0-0-6-9 \mathrm{~Hz}$ after two iterations, $0-0-9-12 \mathrm{~Hz}$ after 74 iterations, and $0-0-12-15 \mathrm{~Hz}$ after one iteration, as shown in Figure 4b. The intensity-norm algorithm can fully use the ultra-low-frequency content in intensity data to avoid cycle skipping even though the original waveform data do not contain low-frequency data of less than $15 \mathrm{~Hz}$. FWI, however, cannot recover the Gaussian-ball anomaly even after 100 iterations (Figure 4c) using data having no information less than $15 \mathrm{~Hz}$. If we use another data set that contains $>10 \mathrm{~Hz}$ information, conventional FWI can successfully invert for the Gaussian-ball velocity anomaly using the frequencies within the $10-15-30-40 \mathrm{~Hz}$ band after 100 iterations (Figure 4d).

\section{Crosswell data}

We consider another, more complicated example for a crosswell data. Illustrated in Figure 5a is a time-domain trace, and in Figure $5 b$ is the corresponding frequency-domain spectrum, in which the frequency contents of less than $150 \mathrm{~Hz}$ have been effectively eliminated. The intensity of the synthetic trace is presented in Figure $5 \mathrm{c}$, and its frequency spectrum is shown in Figure 5d. Comparing the spectra in Figure 5b and 5d, the intensity data have more low-frequency information than the original waveform. The intensity calculation apparently redistributes the wave spectrum into the low- and high-frequency parts. Figure 6a shows the true velocity model, which is $120 \mathrm{~m}$ wide and $300 \mathrm{~m}$ deep and is discretized using a grid interval of $1 \mathrm{~m}$ in the horizontal and vertical directions. The velocity varies from 4390 to $6160 \mathrm{~m} / \mathrm{s}$. There are 76 sources vertically located at $x=10 \mathrm{~m}$, and 76 receivers vertically located at $x=110 \mathrm{~m}$, both arrays extending from $z=50$ to $275 \mathrm{~m}$ with a vertical spacing of $3 \mathrm{~m}$. The synthetic a)

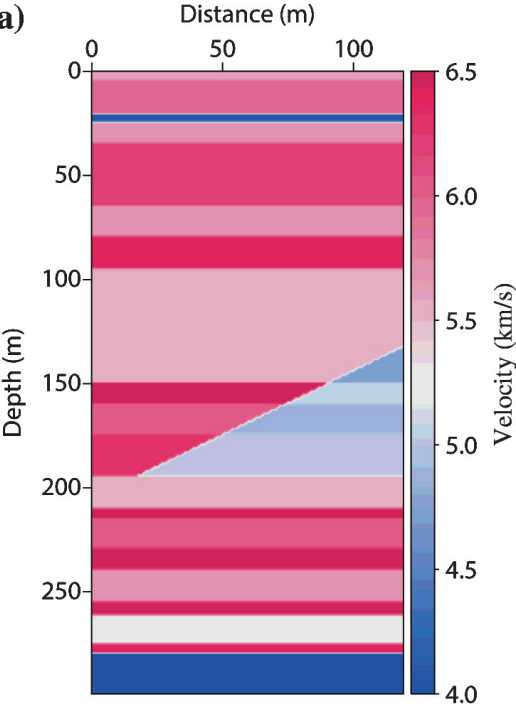

c)

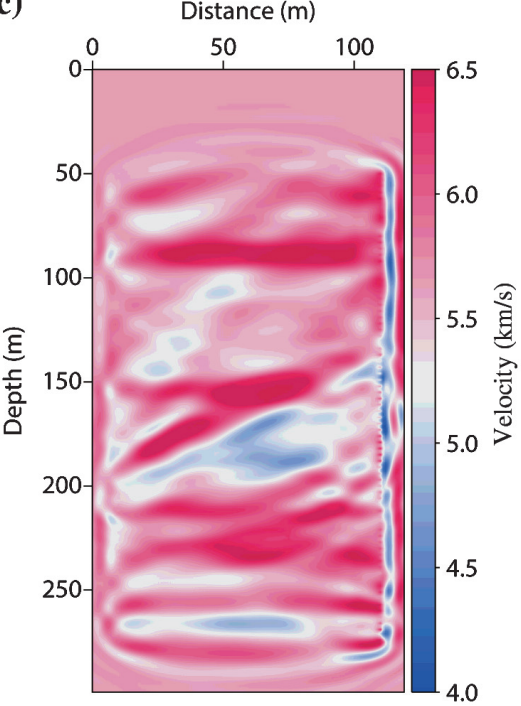

b)

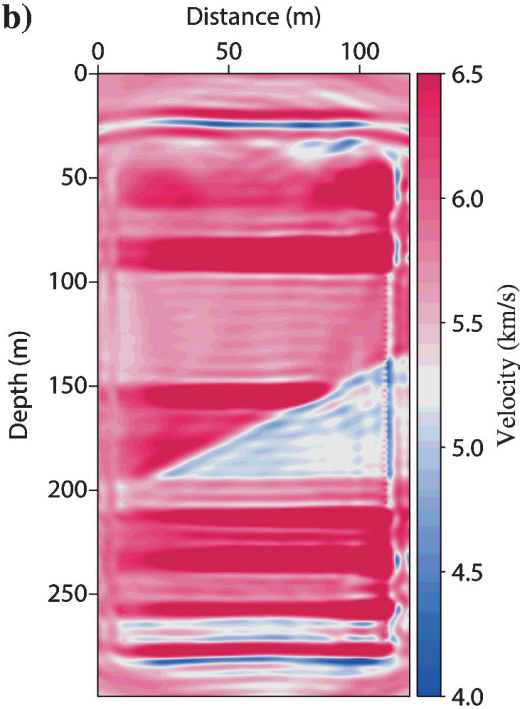

d)

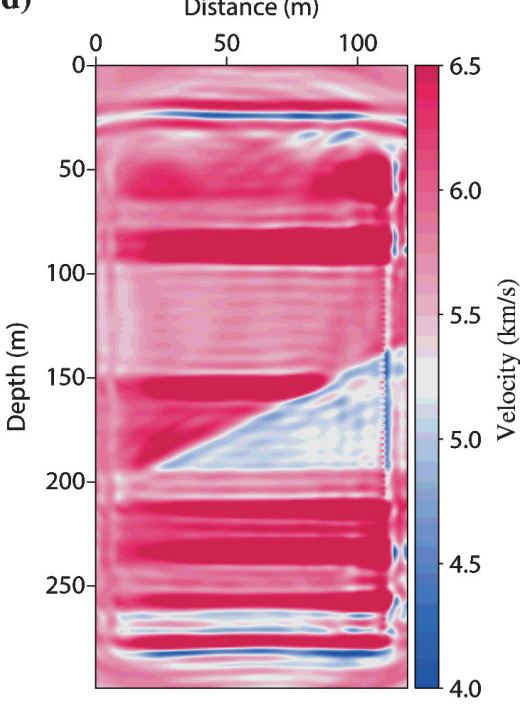

Figure 6. Inverted results using a constant initial velocity $(5490 \mathrm{~m} / \mathrm{s})$. (a) True velocity model, (b) inverted model by conventional FWI without using the multiscale procedure used after 100 iterations, (c) inverted model using $0-0-50-100 \mathrm{~Hz}$ data after one iteration FIWI, then using $0-0-100-150 \mathrm{~Hz}$ data after five iteration FIWIs, and in the frequency band $0-0-150-200 \mathrm{~Hz}$ after one iteration of FIWI, and (d) the FWI inverted model using (c) as the initial model after 100 iterations with the multiscale inversion. 
data set is generated using a 2D full-wave finite-difference code with a 16th-order accuracy in space and a second-order accuracy in time. To guarantee that there is no low-frequency information in the synthetic data, a Butterworth wavelet (e.g., Zhou and McMechan, 1999) with parameters $150-200-400-450 \mathrm{~Hz}$ is used as the source time function. We will use the low-frequency part to implement the FIWI. Two initial models with different constant velocities are used to test how the FIWI can tolerate errors in the initial model. We first use an initial model, which has a constant velocity of $5490 \mathrm{~m} / \mathrm{s}$, and calculate for 100 iterations using conventional FWI. The result is shown in Figure 6b. As a comparison, the FIWI is applied to the same initial model and the intensity data. We apply the a)

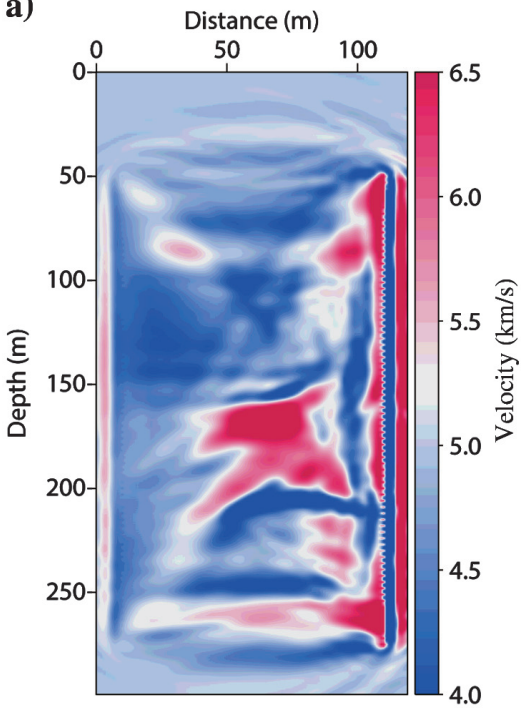

c)

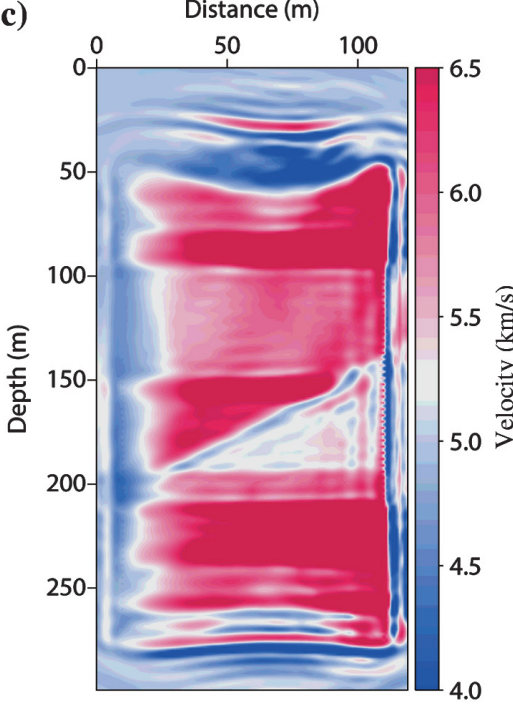

b)

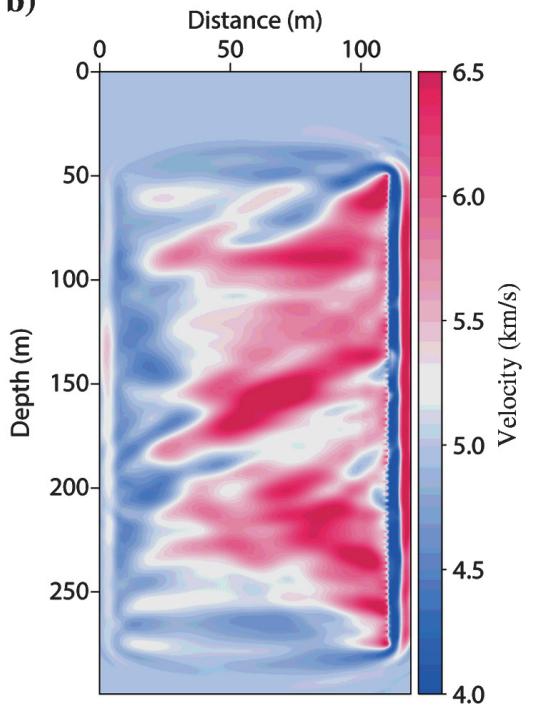

d)

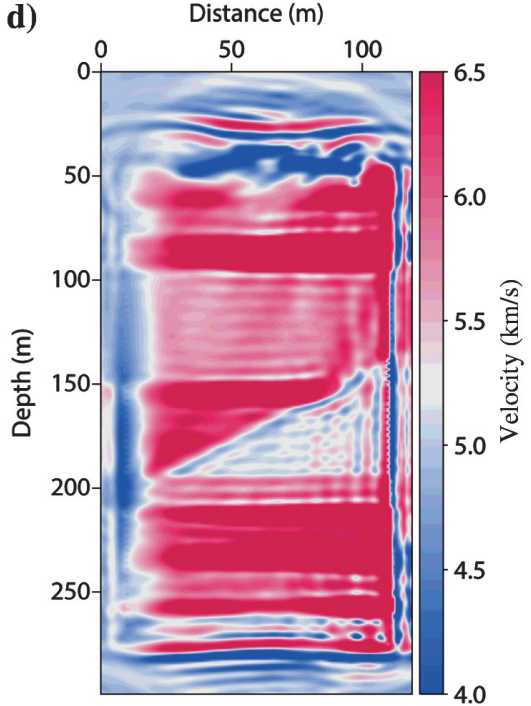

Figure 7. Inverted results using a constant initial velocity $(5000 \mathrm{~m} / \mathrm{s})$. (a) FWI inverted velocity models cannot converge after nine iterations with a $5000 \mathrm{~m} / \mathrm{s}$ constant initial velocity without the multiscale procedure being used, (b) FIWI inverted velocity with a $5000 \mathrm{~m} / \mathrm{s}$ homogeneous initial velocity using multiscale frequencies ranging from $0-0$ $20-50 \mathrm{~Hz}$ for four iterations, $0-0-40-80$ for two iterations, and $0-0-60-120 \mathrm{~Hz}$ for three iterations, and (c and d) FWI (without multiscale being used) inverted velocity models with the initial velocity model in (b) by FIWI after 30 and 100 iterations, respectively.
Butterworth filter to the intensity data from low to high frequencies: at $0-0-50-100 \mathrm{~Hz}$ for one iteration, $0-0-100-150 \mathrm{~Hz}$ for five iterations, and $0-0-150-200 \mathrm{~Hz}$ for one iteration. The result is shown in Figure $6 \mathrm{c}$. The model is then used as the starting model for the conventional FWI for additional 100 iterations, and the result is shown in Fure $6 \mathrm{~d}$. The consistency of Figure $6 \mathrm{a}, 6 \mathrm{~b}$, and $6 \mathrm{~d}$ demonstrate $5490 \mathrm{~m} / \mathrm{s}$, conventional FWI and FIWI + FWI inversion schemes can properly obtain the true velocity model.

Next, we replace the above initial velocity model by a more chaling model, which has a constant velocity of $5000 \mathrm{~m} / \mathrm{s}$. This new initial model deviates more from the true velocity model, and it is expected to cause more severe cycleskipping problems. Under this circumstance, conventional FWI with the same data does not converge at all so that the update stops at the ninth iteration under the line-search condition. This result is shown in Figure 7a. On the other hand, multiscale FIWI after the following processing, at $0-0-20-50 \mathrm{~Hz}$ for four iterations, $0-0-40-80 \mathrm{~Hz}$ for two iterations, and $0-0-60$ $120 \mathrm{~Hz}$ for three iterations, obtained a better result (Figure 7b). Using this inverted model (Figure $7 \mathrm{~b}$ ) as the starting model, we then use conventional FWI for further updating. The FWI results after 30 and 100 iterations are shown in Figure $7 \mathrm{c}$ and $7 \mathrm{~d}$, respectively, in which most of the details in the original model are recovered. This demonstrates that FIWI can largely avoid cycle skipping and provide unbiased low-wavenumber components of the model even in an initial model with large errors.

\section{Marmousi model}

In the third example, we test FIWI using part of the 2D Marmousi model, which is extracted from the original Marmousi model. The model consists of 661 (horizontal) by 201 (vertical) grid points. A grid spacing of $10 \mathrm{~m}$ is used in the horizontal and vertical directions. The acquisition system includes 56 sources, evenly spaced at $120 \mathrm{~m}$ on the top of the model. The source time function is a $10 \mathrm{~Hz}$ Ricker wavelet. There are 661 receivers on the top of the model at an interval of $10 \mathrm{~m}$. A full-wave synthetic data set is calculated from the Marmousi model and the above-mentioned acquisition geometry. Eliminating the low-frequency contents from the synthetic data set to test the validity of FIWI, the data are filtered using a Butterworth filter with a 5-7-9$12 \mathrm{~Hz}$ passband. A representative trace is shown in Figure 8, in which Figure 8a is the time-domain waveform, Figure $8 \mathrm{~b}$ is the frequency spectrum with frequency contents of less than $5 \mathrm{~Hz}$ removed, Figure $8 \mathrm{c}$ is the intensity of the trace, and Figure $8 \mathrm{~d}$ shows the frequency spectrum of the intensity. Comparing Figure $8 \mathrm{~b}$ with Figure $8 \mathrm{~d}$, we see that the latter contains lower frequencies. 
To test the capability of the FIWI, we conduct inversions using two different workflows. In the first one, we use a 1D initial model and the conventional multiscale FWI, in which the velocity linearly increases from 1500 at the top to $3500 \mathrm{~m} / \mathrm{s}$ at the bottom (Figure $9 \mathrm{~b}$ ). To conduct multiscale FWI, the data are band-pass filtered at 5-5.5, 5-6.5, 5-8, 5-10,5-12, and 5-15 $\mathrm{Hz}$ and are sequentially used in the FWI with 180 iterations. The final FWI result is shown in Figure 9c. Compared with Figure 9a, Figure 9c provides certain fine structures. However, the inverted velocity does not match the general trend of the Marmousi model, particularly the high velocity at the deeper part and the shallow upper left part. For this area ranging from 1.30 to $2.0 \mathrm{~km}$ in depth and 2.5 to $5.5 \mathrm{~km}$ in the horizontal direction, the true velocity structure is not recovered. The main reason is the lack of low-frequency data at less than $5 \mathrm{~Hz}$, which leads to cycle skipping.

In the second workflow, we first use the Butterworth filter to filter the intensity and obtain a $0-5 \mathrm{~Hz}$ intensity data set. Then, we apply the multiscale FIWI in $0-2$ and $0-5 \mathrm{~Hz}$ frequency bands using the initial model and the filtered intensity data, and we iterate the FIWI 50 times. Because the intensity data contain very-low-frequency components (refer to Figure 8c and 8d), FIWI produces an inverted model, which is of low spatial resolution but has the correct longwavelength component (Figure 9d). We then use this model as the starting model for conventional FWI. The rest of the workflow is the same as the workflow applied using only the initial model. The final model obtained by the joint application of FIWI and then FWI denoted as FIWI + FWI is shown in Figure 9e. Comparing Figure 9e with $9 \mathrm{c}$, the result of Figure $9 \mathrm{e}$ is very close to the true model (Figure 9a), particular the region at the left from 0 to $2.2 \mathrm{~km}$ in the horizontal direction and the lower middle area, where conventional FWI cannot update well.

In addition, we also extracted velocities along a depth profile at the middle on the surface (Figure 10). In Figure 10a, we compare the true velocity model (solid), the initial model (dashed), and the result obtained using conventional FWI (dotted). In Figure 10b, we compare the intermediate model from FIWI (dashed), and the final result using FIWI plus FWI (dotted). By investigating Figures 9 and 10, the inverted results using joint FIWI and FWI inversions reconstruct the original structure very well. From the surface to the 1.0 depth, the curve of the velocity updated

8. For the Marmousi model: (a) a time-domain trace, (b) the frequency spect of the trace with its frequency contents of less than $5 \mathrm{~Hz}$ have been removed, (c) the intensity of the trace, and (d) the frequency spectrum of the intensity.
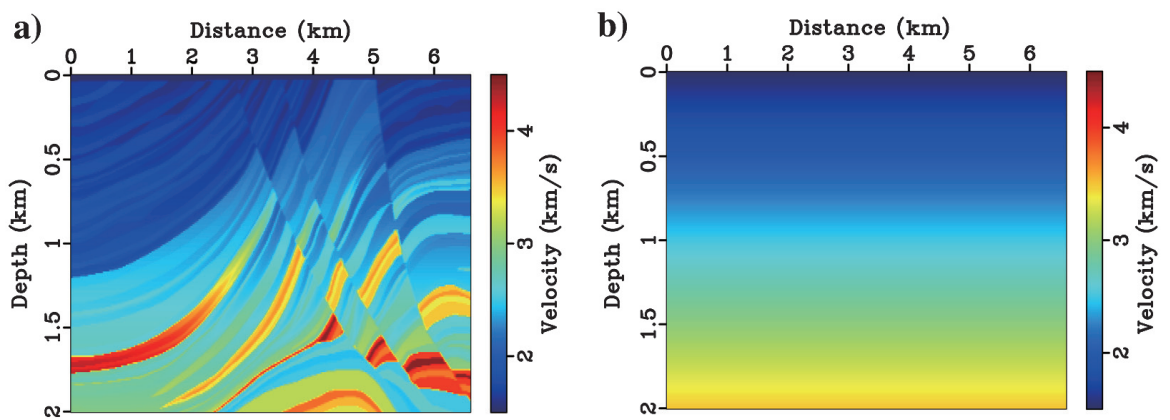

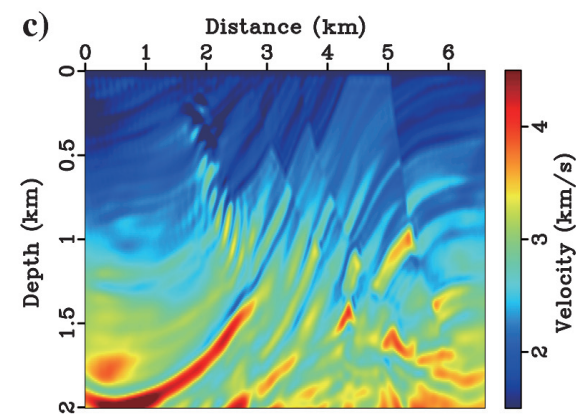

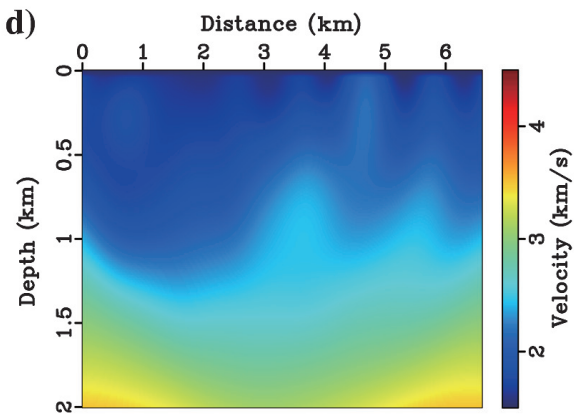

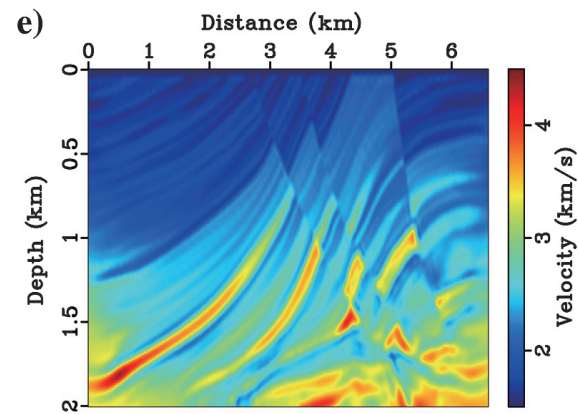

Figure 9. (a) The exact Marmousi velocity model. (b) The initial velocity model for inversion. (c) The inverted velocity using the conventional FWI. (d) The inverted velocity using FIWI. (e) The inverted velocity using FIWI + FWI. 

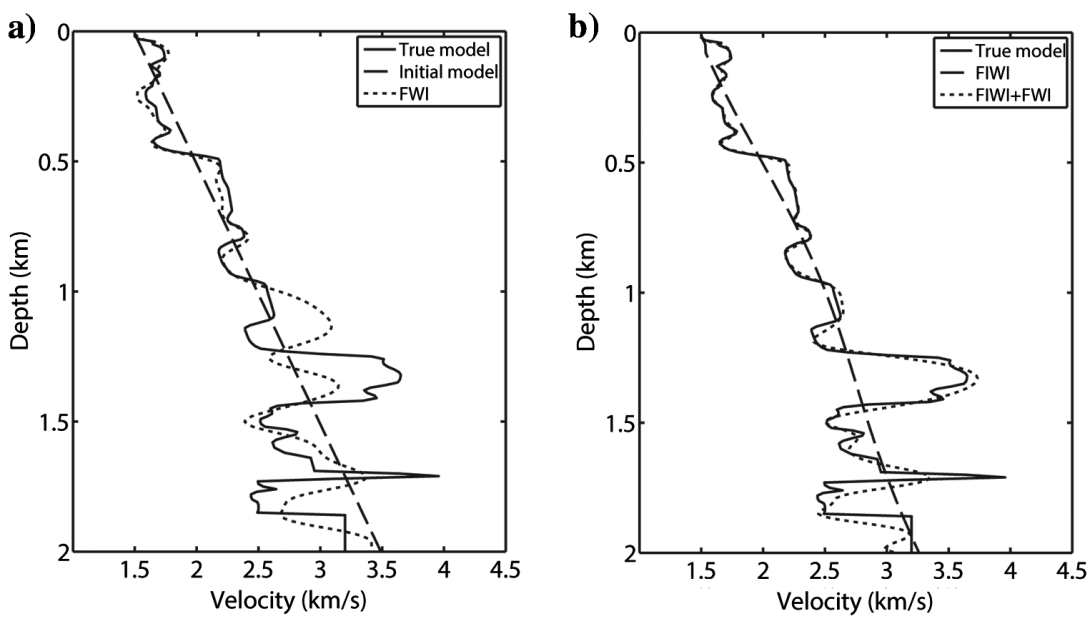

Figure 10. The comparison of the vertical velocity profiles extracted at the middle of the surface using multiscale FWI and multiscale FIWI + FWI, respectively. (a) The FWI result displays better matching from the surface to approximately $1.0 \mathrm{~km}$; however, it starts deviating from the true model when the depth increases. (b) The inverted velocity model (dotted line) using multiscale FIWI + FWI highly overlaps with the true model; other than that, the amplitude at a depth of approximately $1.75 \mathrm{~km}$ is small. a)

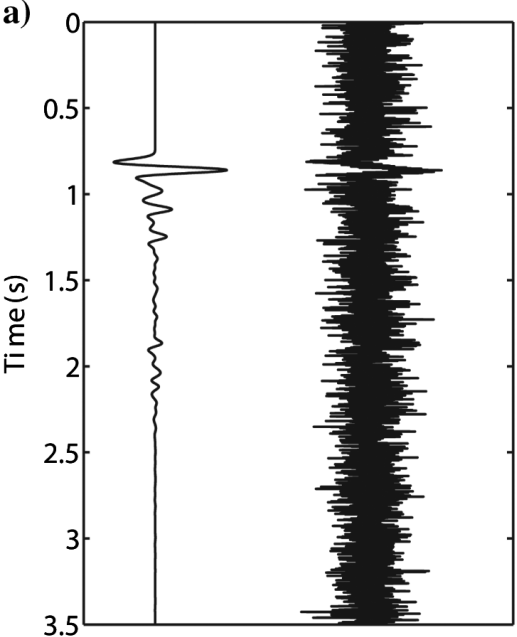

b)

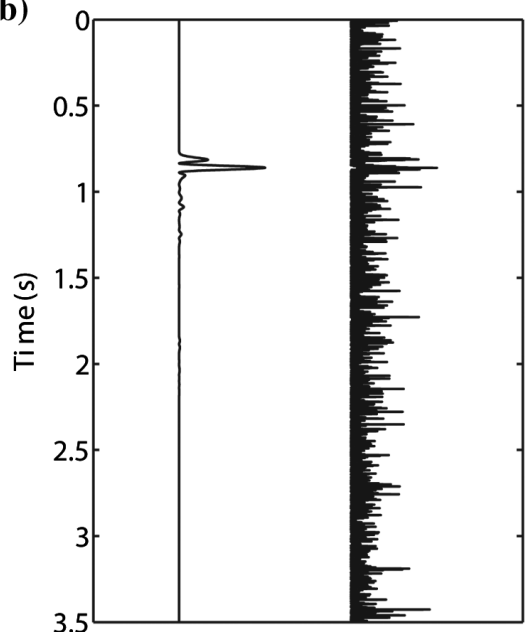

Figure 11. Comparison of a seismic trace computed from the Marmousi model with and without noise. (a) Left: original trace without noise and right: trace with $20 \mathrm{~dB}$ white Gaussian noise. (b) Left: corresponding intensity trace without noise and right: intensity trace with noise.
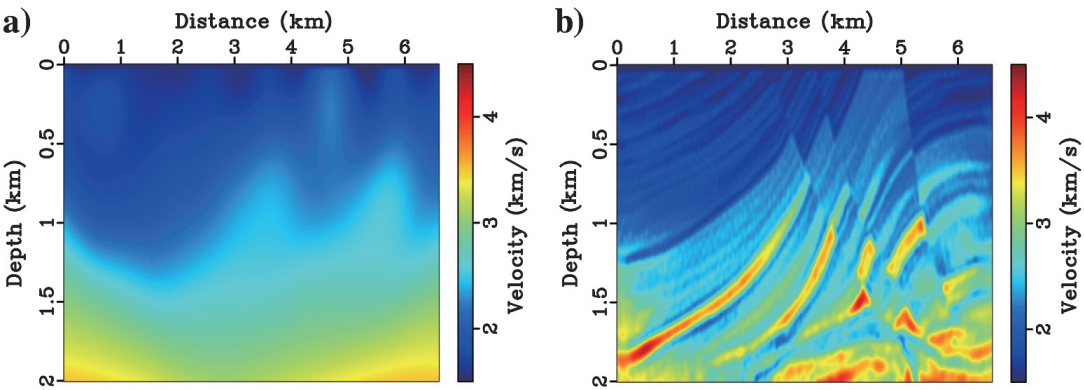

Figure 12. Inversion results using data with $20 \mathrm{~dB}$ Gaussian noise: (a) the inverted result using FIWI and (b) the inverted result using FIWI + FWI. by FIWI + FWI almost completely overlaps the one of the true model and the curve of less than the 1.0 to the $2 \mathrm{~km}$ only have very little delay (deeper) than the true model. It indicates that using the more accurate inverted intermediate velocity model generated by FIWI as the starting model, the final result provides more balanced velocity values for the shallow and the deeper part of the model. For example, in Figure 9a, there are layered dipping structures in the Marmousi model. The FIWI plus FWI method properly reproduces these layers in Figures $9 \mathrm{e}$ and 10b. However, conventional FWI fails to generate these structures in Figure 9c.

To test the performance of FIWI with noisy data, we added white Gaussian noise with $20 \mathrm{~dB}$ signal-to-noise ratio to the time-domain data calculated in the Marmousi model. Sample traces with and without noise are shown in Figure 11. The acquisition geometry, initial model, and the other parameters are the same as those used in the noise-free case. The inverted result using FIWI and the final result using FIWI plus FWI are illustrated in Figure 12a and 12b, respectively. Compared with the noise-free results shown in Figure 9e, Figure 12b is a little blurrier but keeps all the structure features such as the dipping layers at the left and the main structure at the deep of the middle. From the above test, it appears the FIWI intensity-based method is insensitive to a moderate-level uncorrelated white Gaussian noise.

\section{CONCLUSION}

The objective of this paper is to show that the intensity can be used to formulate a new seismic inversion method. Intensity emphasizes the part of the seismic data, in which constructive and destructive interferences occur. The intensity operation redistributes the waveform spectral contents to low- and high-frequency bands. The presence of the low-frequency content in intensity can mitigate the cycle-skipping challenge.

The proposed FIWI approach has been successful in inverting for the subsurface models in the crosswell transmission geometry and the reflection geometry using the Marmousi model. FIWI can effectively mitigate the local-minima problem frequently encountered in traditional FWI when using the data without low-frequency content.

In FIWI, the adjoint source is composed of the intensity differences between the modeled and observed waveform data, multiplied by the modeled waveform data. FIWI preserves the polarity information if the modeled data can be properly normalized associated with observed data, FIWI can still work robustly even when the data contain significant noise. 
The adjoint source of FIWI is still kept in its original data frequency band, which means we do not back extrapolate a low-frequency adjoint source rather than the source with the same frequency-band level as the original data have. The FIWI can set up relationships between the data frequency and model wavenumber in the context of intensity.

This characteristic will physically keep the wave propagation ignited by the FIWI adjoint source reflected by the response of media.

Finally, the presence of the low-frequency intensity data in the inversion can help to avoid cycle skipping. Hence, FIWI reduces the tendency for the iterative algorithm to be trapped in local minima. Compared with the conventional FWI, FIWI has a weaker dependence on the accuracy of the initial model and low-frequency components in the data. Numerical tests using synthetic data demonstrate that, even starting from an initial model having large velocity errors compared with the true model, FIWI is still able to recover large-scale velocity variations satisfactorily.

\section{ACKNOWLEDGMENTS}

We thank S. Xu, H. Zhou, Y. Luo, G. T. Schuster, and M. Lu for their helpful suggestions and insightful comments. The research was partially funded by Statoil Petroleum (grant no. 4503288025), the National Nature Science Foundation of China (grant nos. 41730425 and 41430321), and The National Oil and Gas Major Project of China (grant no. 2017ZX05008-007).

\section{DATA AND MATERIALS AVAILABILITY}

Data associated with this research are available and can be obtained by contacting the corresponding author.

\section{APPENDIX A}

\section{THE GRADIENT OF NORMALIZED FULL INTENSITY OBJECTIVE}

The objective function using the normalized intensity is

$$
E(\mathbf{m})=\frac{1}{4} \sum_{s} \sum_{r} \int_{0}^{T} d I_{\text {norm }}\left(\mathbf{x}_{r}, t ; \mathbf{x}_{s}\right)^{2} d t,
$$

where

$$
\begin{gathered}
d I_{\text {norm }}\left(\mathbf{x}_{r}, \mathbf{x}_{s}, t\right)=\frac{d_{c}^{\dagger}\left(\mathbf{x}_{r}, t ; \mathbf{x}_{s}\right) d_{c}\left(\mathbf{x}_{r}, t ; \mathbf{x}_{s}\right)}{\bar{P}_{c}\left(\mathbf{x}_{r} ; \mathbf{x}_{s}\right)} \\
-\frac{d_{o}^{\dagger}\left(\mathbf{x}_{r}, t ; \mathbf{x}_{s}\right) d_{o}\left(\mathbf{x}_{r}, t ; \mathbf{x}_{s}\right)}{\bar{P}_{0}\left(\mathbf{x}_{r} ; \mathbf{x}_{s}\right)} .
\end{gathered}
$$

A slowness perturbation of equation A-1 is given using by minimizing the objective function

$$
\begin{gathered}
\delta \mathbf{m}=\frac{\partial E(\mathbf{m})}{\partial \mathbf{m}}=\frac{1}{2} \sum_{s} \sum_{r} \int_{0}^{T} d I_{\text {norm }}\left(\mathbf{x}_{r}, t ; \mathbf{x}_{s}\right) \frac{\partial d I_{\text {norm }}\left(\mathbf{x}_{r}, t ; \mathbf{x}_{s}\right)}{\partial m} d t, \\
=\frac{1}{2} \sum_{s} \sum_{r} \int_{0}^{T} d I_{\text {norm }}\left(\mathbf{x}_{r}, t ; \mathbf{x}_{s}\right) \frac{\partial}{\partial m}\left(\frac{d_{c}^{2}\left(\mathbf{x}_{r}, t ; \mathbf{x}_{s}\right)}{\bar{P}_{c}\left(\mathbf{x}_{r} ; \mathbf{x}_{s}\right)}\right) d t .
\end{gathered}
$$

The derivative of normalized intensity with respect to media parameter is

$$
\frac{\partial}{\partial m}\left(\frac{d_{c}^{2}\left(\mathbf{x}_{r}, t ; \mathbf{x}_{s}\right)}{\bar{P}_{c}\left(\mathbf{x}_{r} ; \mathbf{x}_{s}\right)}\right)=2 A+2 B,
$$

where

$$
\begin{aligned}
& A=\frac{\left[\frac{\partial d_{c}\left(\mathbf{x}_{r}, t ; \mathbf{x}_{s}\right)}{\partial m}\right] d_{c}\left(\mathbf{x}_{r}, t ; \mathbf{x}_{s}\right)}{\bar{P}_{c}\left(\mathbf{x}_{r} ; \mathbf{x}_{s}\right)}, \\
& B=-\frac{d_{c}^{2}\left(\mathbf{x}_{r}, t ; \mathbf{x}_{s}\right) \frac{1}{T} \int_{\tau=0}^{T}\left[\frac{\partial d_{c}\left(\mathbf{x}_{r}, \tau ; \mathbf{x}_{s}\right)}{\partial m}\right] d_{c}\left(\mathbf{x}_{r}, \tau ; \mathbf{x}_{s}\right) d \tau}{\bar{P}_{c}\left(\mathbf{x}_{r} ; \mathbf{x}_{s}\right)^{2}} .
\end{aligned}
$$

Substituting equation A-4 to equation A-3, we have the FIWI gradient

$$
\begin{aligned}
& \delta m=\frac{\partial E(m)}{\partial m}=\sum_{s} \sum_{r} \int_{t=0}^{T} d I_{\mathrm{norm}}\left(\mathbf{x}_{r}, \mathbf{x}_{s}, t\right)(A+B) d t \\
& =\sum_{s} \sum_{r}\left[\int_{t=0}^{T} \frac{d I_{\mathrm{norm}}\left(\mathbf{x}_{r}, t ; \mathbf{x}_{s}\right) d_{c}\left(\mathbf{x}_{r}, t ; \mathbf{x}_{s}\right)}{\bar{P}_{c}\left(\mathbf{x}_{r} ; \mathbf{x}_{s}\right)} \frac{\partial d_{c}\left(\mathbf{x}_{r}, t ; \mathbf{x}_{s}\right)}{\partial m} d t\right. \\
& -\frac{1}{T} \int_{t=0}^{T} \frac{d I_{\text {norm }}\left(\mathbf{x}_{r}, t ; \mathbf{x}_{s}\right) d_{c}^{2}\left(\mathbf{x}_{r}, t ; \mathbf{x}_{s}\right)}{\bar{P}_{c}\left(\mathbf{x}_{r} ; \mathbf{x}_{s}\right)^{2}} \\
& \left.\quad \times \int_{\tau=0}^{T}\left(\frac{\partial d_{c}\left(\mathbf{x}_{r}, \tau ; \mathbf{x}_{s}\right)}{\partial m}\right) d_{c}\left(\mathbf{x}_{r}, \tau ; \mathbf{x}_{s}\right) d \tau d t\right] .
\end{aligned}
$$

We exchange variable names $t$ with $\tau$, and reorganize and obtain

$$
\begin{aligned}
& \delta \mathbf{m}=\frac{\partial E(\mathbf{m})}{\partial \mathbf{m}}, \\
& =\sum_{s} \sum_{r}\left[\int_{0}^{T} \frac{d I_{\text {norm }}\left(\mathbf{x}_{r}, t ; \mathbf{x}_{s}\right) d_{c}\left(\mathbf{x}_{r}, t ; \mathbf{x}_{s}\right)}{\bar{P}_{c}\left(\mathbf{x}_{r} ; \mathbf{x}_{s}\right)} \frac{\partial d_{c}\left(\mathbf{x}_{r}, t ; \mathbf{x}_{s}\right)}{\partial m} d t\right. \\
& \left.-\frac{\frac{1}{T} \int_{t=0}^{T} d I_{\text {norm }}\left(\mathbf{x}_{r}, t ; \mathbf{x}_{s}\right) d_{c}^{2}\left(\mathbf{x}_{r}, t ; \mathbf{x}_{s}\right) d t \int_{\tau=0}^{T}\left(\frac{\partial d_{c}\left(\mathbf{x}_{r}, \tau ; \mathbf{x}_{s}\right)}{\partial m}\right) d_{c}\left(\mathbf{x}_{r}, \tau ; \mathbf{x}_{s}\right) d \tau}{\bar{P}_{c}\left(\mathbf{x}_{r} ; \mathbf{x}_{s}\right)^{2}}\right], \\
& =\sum_{s} \sum_{r}\left[\int_{0}^{T} \frac{d I_{\text {norm }}\left(\mathbf{x}_{r}, t ; \mathbf{x}_{s}\right) d_{c}\left(\mathbf{x}_{r}, t ; \mathbf{x}_{s}\right)}{\bar{P}_{c}\left(\mathbf{x}_{r} ; \mathbf{x}_{s}\right)} \frac{\partial d_{c}\left(\mathbf{x}_{r}, t ; \mathbf{x}_{s}\right)}{\partial m} d t\right. \\
& \left.-\frac{\frac{1}{T} \int_{\tau=0}^{T} d I_{\text {norm }}\left(\mathbf{x}_{r}, \tau ; \mathbf{x}_{s}\right) d_{c}^{2}\left(\mathbf{x}_{r}, \tau ; \mathbf{x}_{s}\right) d \tau \int_{t=0}^{T} \frac{\partial d_{c}\left(\mathbf{x}_{r}, t ; \mathbf{x}_{s}\right)}{\partial m} d_{c}\left(\mathbf{x}_{r}, t ; \mathbf{x}_{s}\right) d t}{\bar{P}_{c}\left(\mathbf{x}_{r} ; \mathbf{x}_{s}\right)^{2}}\right], \\
& =\sum_{s} \sum_{r}\left[\int_{0}^{T} \frac{d I_{\text {norm }}\left(\mathbf{x}_{r}, t ; \mathbf{x}_{s}\right) d_{c}\left(\mathbf{x}_{r}, t ; \mathbf{x}_{s}\right)}{\bar{P}_{c}\left(\mathbf{x}_{r} ; \mathbf{x}_{s}\right)} \frac{\partial d_{c}\left(\mathbf{x}_{r}, t ; \mathbf{x}_{s}\right)}{\partial m} d t\right. \\
& \left.-\frac{\frac{1}{T} \int_{\tau=0}^{T} d I_{\text {norm }}\left(\mathbf{x}_{r}, \tau ; \mathbf{x}_{s}\right) d_{c}^{2}\left(\mathbf{x}_{r}, \tau ; \mathbf{x}_{s}\right) d \tau}{\bar{P}_{c}\left(\mathbf{x}_{r} ; \mathbf{x}_{s}\right)} \int_{t=0}^{T} \frac{d_{c}\left(\mathbf{x}_{r}, t ; \mathbf{x}_{s}\right)}{\bar{P}_{c}\left(\mathbf{x}_{r} ; \mathbf{x}_{s}\right)} \frac{\partial d_{c}\left(\mathbf{x}_{r}, t ; \mathbf{x}_{s}\right)}{\partial m} d t\right], \\
& =\sum_{s} \sum_{r}\left\{\int_{0}^{T}\left[d I_{\text {norm }}\left(\mathbf{x}_{r}, t ; \mathbf{x}_{s}\right)-\frac{\frac{1}{T} \int_{\tau=0}^{T} d I_{\text {norm }}\left(\mathbf{x}_{r}, \tau ; \mathbf{x}_{s}\right) d_{c}^{2}\left(\mathbf{x}_{r}, \tau ; \mathbf{x}_{s}\right) d \tau}{\bar{P}_{c}\left(\mathbf{x}_{r} ; \mathbf{x}_{s}\right)}\right]\right. \\
& \left.\times \frac{d_{c}\left(\mathbf{x}_{r}, t ; \mathbf{x}_{s}\right)}{\bar{P}_{c}\left(\mathbf{x}_{r} ; \mathbf{x}_{s}\right)} \frac{\partial d_{c}\left(\mathbf{x}_{r}, t ; \mathbf{x}_{s}\right)}{\partial m} d t\right\} \text {. }
\end{aligned}
$$

\section{REFERENCES}

Alkhalifah, T., and Y. Choi, 2014, From tomography to FWI with a single objective function: Geophysics, 79, no. 2, R55-R61, doi: 10.1190/ geo2013-0291.1. 
Alkhalifah, T., and Z. Wu, 2015, Multi-scattering inversion for low model wave numbers: 85th Annual International Meeting, SEG, Expanded Abstracts, $1165-1170$.

Almomin, A., and B. Biondi, 2012, Tomographic full-waveform inversion: Practical and computationally feasible approach: 82nd Annual International Meeting, SEG, Expanded Abstracts, doi: 10.1190/segam2012-0976.1.

Baeten, G., J. W. de Maag, R. E. Plessix, M. Klaassen, T. Qureshi, M. Kleemeyer, F. T. Kroode, and R. Zhang, 2013, The use of the low frequencies in a full waveform inversion and impedance inversion land seismic case study: Geophysical Prospecting, 61, 701-711, doi: 10.1111/1365-2478 .12010 .

Bharadwj, P., W. Mulder, and G. Drijkoningen, 2016, Full waveform inversion with an auxiliary bump functional: Geophysical Journal International, 206, 1076-1092.

Biondi, B., and A. Almomin, 2013, Tomographic full waveform inversion (TFWI) by extending the velocity model along the time-lag axis: 83rd Annual International Meeting, SEG, Expanded Abstracts, 1031-1034.

Born, M., and E. Wolf, 1999, Principles of optics, 7th ed.: Cambridge University Press.

Bozdag, E., J. Trampert, and J. Tromp, 2011, Misfit functions for full waveform inversion based on instantaneous phase and envelope measurements: Geophysical Journal International, 185, 845-870.

Brittan, J., J. Bai, H. Delome, C. Wang, and D. Yingst, 2013, Full waveform inversion: The state of the art: First Break, 31, 75-81.

Bunks, C., F. M. Saleck, S. Zaleski, and G. Chavent, 1995, Multiscale seismic waveform inversion: Geophysics, 60, 1457-1473, doi: 10.1190/1 .1443880 .

Choi, Y., and T. Alkhalifah, 2017, Full waveform inversion using an energybased objective function with efficient calculation of the Gradient: 79th Annual International Conference and Exhibition, EAGE, Extended Abstracts, doi: 10.3997/2214-4609.201701343.

Clément, F., G. Chavent, and S. Gómez, 2001, Migration-based traveltime waveform inversion of 2-D simple structures: A synthetic example: Geophysics, 66, 845-860, doi: 10.1190/1.1444974.

Lailly, P., 1983, The seismic inverse problem as a sequence of before stack migrations: Conference on Inverse Scattering, Theory and Application, SIAM, Expanded Abstracts, 206-220.

Leeuwen, T. V., and W. A. Mulder, 2010, A correlation-based misfit criterion for wave-equation traveltime tomography: Geophysical Journal International, 182, 1383-1394, doi: 10.1111/j.1365-246X.2010.04681.x.

Liu, Z., and Y. Zheng, 2015, Direct waveform inversion: 85th Annual International Meeting, SEG, Expanded Abstracts, 1268-1273.

Luo, J., and R. Wu, 2015, Seismic envelop inversion: Reduction of local minima and noise resistance: Geophysical Prospecting, 63, 597-614, doi: 10.1111/1365-2478.12208.

Luo, Y., Y. Ma, Y. Wu, H. Liu, and L. Cao, 2016, Full traveltime inversion: Geophysics, 81, no. 5, R261-R274, doi: 10.1190/geo2015-0353.1.

Luo, Y., and G. T. Schuster, 1991, Wave-equation traveltime inversion: Geophysics, 56, 645-653, doi: 10.10.1190/1.1443081.

Ma, Y., and D. Hale, 2013, Wave-equation reflection traveltime inversion with dynamic warping and full-waveform inversion: Geophysics, 78 , no. 6, R223-R233, doi: 10.1190/geo2013-0004.1.
Plessix, R., G. Baeten, J. W. de Maag, M. Klaassen, R. Zhang, and Z. Tao, 2010, Application of acoustic full waveform inversion to a low-frequency large-offset land data set: 80th Annual International Meeting, SEG, Expanded Abstracts, 930-934.

Pratt, R. G., 1999, Seismic waveform inversion in the frequency domain. Part I: Theory and verification in a physical scale model: Geophysics, 64, 888-901, doi: 10.1190/1.1444597.

Ravaut, C., S. Operto, L. Improta, J. Virieux, A. Herrero, and P. D. Aversana, 2004, Multiscale imaging of complex structures from multifold wideaperture seismic data by frequency-domain full-waveform tomography: Application to a thrust belt: Geophysical Journal International, 159, 1032-1056, doi: 10.1111/j.1365-246X.2004.02442.x.

Shen, P., and W. W. Symes, 2008, Automatic velocity analysis via shot profile migration: Geophysics, 73, no. 5, VE49-VE59, doi: 10.1190/1 .2972021

Shin, C., and Y. H. Cha, 2009, Waveform inversion in the Laplace-Fourier domain: Geophysical Journal International, 177, 1067-1079, doi: 10 $.1111 / \mathrm{j} .1365-246 X .2009 .04102 . x$.

Sirgue, L., and R. G. Pratt, 2004, Efficient waveform inversion and imaging: A strategy for selecting temporal frequencies: Geophysics, 69, 231-248, doi: 10.1190/1.1649391.

Tarantola, A., 1984, Inversion of seismic reflection data in the acoustic approximation: Geophysics, 49, 1259-1266, doi: 10.1190/1.1441754.

Tarantola, A., 2005, Inverse problem theory and methods for model parameter estimation: SIAM.

Virieux, J., and S. Operto, 2009, An overview of full waveform inversion in exploration geophysics: Geophysics, 74, no. 6, WCC1-WCC26, doi: 10 $.1190 / 1.3238367$

Wu, R. S., J. Luo, and B. Wu, 2014, Seismic envelop inversion and modulation signal model: Geophysics, 79, no. 3, WA13-WA24, doi: 10.1190/ geo2013-0294.1.

Wu, R. S., and Y. Zheng, 2014, No-linear partial derivative and its De Wolf approximation for non-linear seismic inversion: Geophysical Journal International, 196, 1827-1843, doi: 10.1093/gji/ggt496.

Xie, X. B., 2015, An angle-domain wavenumber filter for multi-scale fullwaveform inversion: 85th Annual International Meeting, SEG, Expanded Abstracts, 1132-1137.

Xu, S., D. Wang, F. Chen, Y. Zhang, and G. Lambare, 2012, Full waveform inversion for reflected seismic data: 74th Annual International Conference and Exhibition, EAGE, Extended Abstracts, doi: 10.3997/2214-4609 .20148725 .

Zhang, S., Y. Luo, and G. Schuster, 2015, Shot- and angle-domain wave-equation traveltime inversion of reflection data: Synthetic and field data examples: Geophysics, 80, no. 4, S79-S92, doi: 10.1190/geo2014-0223.1.

Zhang, Y., and D. Wang, 2009, Traveltime information-based wave-equation inversion: Geophysics, 74, no. 6, WCC27-WCC36, doi: 10.1190/1.3243073.

Zhou, H., L. Amundsen, and G. Zhang, 2012, Fundamental issues in full waveform inversion: 82nd Annual International Meeting, SEG, Expanded Abstracts, doi: 10.1190/segam2012-0878.1.

Zhou, H., and G. A. McMechan, 1999, Parallel Butterworth and Chebshev dip filters with applications to 3-D seismic migration: Geophysics, 64, 1573-1578, doi: $10.1190 / 1.1444661$. 